\title{
Improving Nurses' Hand-off Process on Oncology Setting Using Lean Management Principles
}

\author{
Omar Ayaad ${ }^{1 *}$, Anas Haroun ${ }^{1}$, Rawya Yaseen ${ }^{1}$, Fouad Thiab ${ }^{2}$, Khalid \\ Al-Rawashdeh ${ }^{2}$, Iqbal Mohammad ${ }^{2}$, Mohammad Aqtash ${ }^{2}$, Saleh Qadumi ${ }^{3}$, Yazan \\ Altantawi $^{3}$, Ahmad Nairat ${ }^{1}$
}

\begin{abstract}
Background: Patients in oncology setting are struggling with the complexed disease, and long and intensive treatment options. This increase the need of patients for more coordination and effective hand-off between health providers including nurses. Aims: The main aim of this project is to improve the effectiveness of hand-off between nurses in the oncology setting using lean management principles. Methods: One group pretest-posttest quasi-experimental design was conducted at King Hussain Cancer Center during quarter two to quarter four in 2017. The project was conducted using the lean tools including root cause analysis, redesigning the hand-off process; using structured tools, and standardization of the hand-off process. Results: The finding of this project showed a significant decreasing in the hand-off duration and the incidence of events related to nursing practice deviation in post-intervention. Moreover, the results showed that the nurse satisfaction score was improved. However, there is a little difference in patient satisfaction results between two quarters for overall satisfaction and per each domain. Conclusion: The project approved that the use of structured tools, safety briefing, and standardized hand-off process play important role in improving the effectiveness of the hand-off process.
\end{abstract}

Keywords: Lean- nurses' hand-off- nurse- oncology setting- hand-off tools

Asian Pac J Cancer Prev, 20 (5), 1563-1570

\section{Introduction}

Nurse hand-off is a transition and acceptance process for patient care responsibility between nurses by utilized effective communication (The Joint Commission, 2014). The Hand-offs between healthcare professionals has been recognized by the Joint Commission as a national patient safety goal, indicating queries primarily as a common cause of errors in healthcare organizations (The Joint Commission, 2017). Moreover, the Agency on Healthcare Research and Quality (AHRQ) recognized enhancing the effectiveness of Hand-Offs among healthcare professions as a high priority in national works to enhance patient safety (AHRQ, 2016)

Furthermore, the Joint Commission expects that all caregivers, including nurses, should execute a standardized way to hand-off process consisting an ability to ask and answer the questions. For these reasons, The Joint Commission National Patient Safety Goals mentioned many vital points to be considered during any hand-off process. These points include: ensuring interactive conversations between nurses; sharing up-to-date and reliable patient' information, limited interruptions; engaging the patient in hand-off process, enhancing the ability of the caregiver to verify and review any relevant historical data (AHRQ, 2016).

There are four common styles of hand-off mentioned in the literature used between nurses which are verbal hand-off; tape recorded hand-off; bedside hand-off; and written hand-off (Anderson et al., 2015; Bakon et al., 2017; Ilan et al., 2012). Many studies showed the ability of the use of more than one style during the hand-off with use of structured tools (Anderson et al., 2015; Ilan et al., 2012)

Despite all mentioned recommendations, many healthcare organizations are complaining of substandard hand-off process due to unstandardized hand-off process between caregivers, missing of critical information during the hand-off; and length of hand-off process. These complaints cause many negative consequences such as delay in care; inadequate treatment, adverse events; increased length of stay, avoidable readmissions, costs, work inefficiency, and other patient harms which have negatively affect patient outcome and caregiver satisfaction including nurses

Patients in oncology setting are struggling with the complexed disease including complicated signs and 
symptoms such as an immobility, decrease in blood counts, electrolyte discrepancies which have significant impacts on their well-being; and various types of long and intensive treatment options such as chemotherapy, radiotherapy, surgeries; and expensive treatment which put the patients and their family in financial crisis (Petersenet al., 2015; Zapka et al., 2012). This increase the patients' needs for more care and support, proper coordination, effective hand-off between health providers including nurses, education, and engagement in their care plan (Gupta et al., 2007; Page et al., 2016; Zapka et al., 2012). For this reasons, the effective hand-off between nurses is considered an essential as treatment management strategy because of its role to safe, effective, appropriate care (Azzani et al., 2014; Gupta et al., 2007).

As an integrated literature review for 45 articles indicated there are many issues for hand-off such as the patient/career engagement, involving the multidisciplinary team, and confidentiality. Moreover, the results identified a lack of literature regarding the changing responsibility and accountability during the hand-off processes and auditing practices. The structured tool was strongly recommended to be used during the hand-off; despite no specific tool was recognized proper for all settings (Anderson et al., 2015). Likewise, a review was conducted in 2016 indicated that there are four main obstacles to efficient hand-offs: communication difficulties, the social environment, language barriers, and the physical environment. Furthermore, there are many strategies to improve hand-offs are available in literature such as standardization of hand-offs; clinical training; and improve environmental factors (Colvin et al., 2016).

Moreover, the utilizing evidence-based process and tools in hand-off process plays important role in reducing hand-off-related problems from $(25.8 \%)$ to $(7.9 \%)(\mathrm{P}<.05)$, increasing compliance to hand-off tools from $86 \%$ to $96 \%(\mathrm{P}<.05)$, ensuring a clear transition of responsibility, decreeing the interruptions during hand-off was improved from $84 \%$ to $90 \%(\mathrm{P}<.05)$, and increasing overall satisfaction with the new hand-off process from $55 \%$ to $70 \%(\mathrm{P}<.05)$ (Bigham et al., 2014).

Likewise, another project aims to increase the transferring of hand-off information without increasing the hand-off duration by conducted a prospective cohort study in the post-anesthesia care unit at a pediatric hospital in Northern California. The intervention consisted of requesting the sending team to involve an anesthesiologist, a surgeon, and a circulating nurse in hand-off, while the receiving team to involve the PACU nurse; using standardized tools for hand-off. Pre and post data was measured. The results showed that the information transfer was increased significantly from $49 \%$ to $83 \%$. Total satisfaction scores were increased from 36 to 44 . Moreover, the duration of the hand-offs was decreased from $4.1 \mathrm{~min}$ to $3.5 \mathrm{~min}$. It was recommended that use a standardized tool, team-based approach to OR-to-PACU hand-offs enhance the effectiveness of hand-off process (Caruso et al., 2015).

A study aimed to identify the role of the use of nurse-led safety debriefing in creating a culture of safety at obstetric emergencies through ensuring standardization, teamwork, and interdisciplinary communication. For this reason, a standard protocol was utilized to be followed in all obstetric emergencies to standardize the staff attendance, guide communication, validate the errors, and identify areas for improvement. Moreover, a formal course was attended by senior nurses on debriefing. The post-implementation data showed ten and more ideas of improvement regarding working process were recognized. $90 \%$ of them have been solved (Tirelli et al., 2017).

Despite a lot of efforts were performed worldwide to improve hand-off between nurses in a different setting. The newly reported studies showed that the hand-off between nurses needs more improvement and is still considered a challenge for health and nurse managers (Colvin et al., 2016; Mardis et al., 2017). Moreover, there are no studies or projects in literature address the needs and improvement of hand-off between nurses in an oncology setting.

Lean management is considered a management system aims to organize the operation design which reduced waste and time-consuming, improved safety and quality, and decreased the costs in health industries by using several tools such as Fishbone, flow chart in addition to redesign the operational work (Costaet al., 2017; D'Andreamatteo et al., 2015). Many health organizations such as National Health Service (NHS) and Institute for Healthcare Improvement (IHI) have recommended utilizing the lean concepts in health setting to enhance work efficiency (Costa et al., 2017; D’Andreamatteo et al., 2015; Machado et al., 2013; Sloan et al., 2014).

The multidisciplinary Lean project was conducted aimed to improve the hand-off regarding patient safety, efficiency, and effectiveness by developing the current process and value stream mapping. Then a new process was proposed using a single, structured, bedside tool for all care providers. A standardized hand-off tool was implemented. The results showed the average wait time declined by 58 minutes, process time declined by 9 minutes, the error rate reduced by 1.3 errors/patient, staff satisfaction increased from $48 \%$ to $73 \%$, and finally, the costs were suspended (Gleich et al., 2015).

In this study, the lean principle was conducted as an intervention to improve the effectiveness of hand-off process between nurses in oncology setting aiming to decrease the time and resource waste, improve the patient and nurse satisfaction, ensuring safe transferring of the patient between shits.

The literature showed that lean healthcare has been growing into a significant strand of research and quality projects in the early 2,000s.it is considered a most common tool used to ensure cost effectiveness and removal of wastes, and to enhance patient and employee satisfaction. (Costa et al., 2017; D'Andreamatteo et al., 2015; Lawal et al., 2014; Sloan et al., 2014).

The findings of the current project would provide the health and nurse managers with a valuable information about how the oncology setting can adopt the lean principles to improve work effectiveness and increase patient and nurse satisfaction, and how the hand-off between nurses can be improved by utilizing lean principle in the oncology setting.

Based on previous studies, there are no studies and 
projects adopt the lean principle to enhance the hand-off between nurses in an oncology setting.

\section{Aim}

The main aim of this project is to improve the effectiveness of hand-off between nurses in oncology setting using lean principles.

\section{Materials and Methods}

\section{Setting}

The project was conducted at the King Hussein Cancer Center (KHCC), a non-governmental, non-profit, specialized center for treating patients with cancer located in Amman, Jordan. The total number of nurses was 500 nurses. The project was conducted at King Hussain Cancer Center during quarter two to quarter four in 2017. This setting was selected because it is the biggest oncology center in Jordan and it's interested to conduct this project.

The project was applied in all inpatient units (VIP, Medical, Medical-Surgical, Pediatric, Palliative, Bone Marrow Transplant, And Leukemia floors), and focused on Hand-off between nurses during shift changes.

The project included members from different nursing areas such as nursing quality and safety (leader) to ensure proper utilizing of lean tools in the project, four nurse managers (members) to facilitate the implementation of the project in the selected units, and two clinical nurses (members) to help in designing the project based on clinical nurses' needs, and sponsored by chief nurse officer to facilitate the application of project in organization-wide.

The hand-off process between nurses during the shift changes was undergone through three phases as Figure 1 shown. The first phase was conducted by coming and going charges nurses at the nursing station. During this period, the critical information about all the patients in the floor was exchanged. The exchange the information during this phase did not depend on standardized tools. The average length of this phase was 10 minutes.

The second phase was conducted at nursing hand-off room where the all coming nurses was setting and waiting for their patients to be endorsed by going nurses who entered the room one by one to endorse all patients to all coming nurses. The exchange the information during this phase did not depend on standardized tools where the going nurses presented and ordered the information regarding their patients according to their views. The average length of this phase was 35 minutes.

The third phase was conducted at the bedside by the coming nurses and the going nurses which includes checking the patient condition and their medication. During this phase, the patient was not engaged in the hand-off process. The average length of this phase was 5 minutes. Figure 1 illustrates the current process analysis.

\section{Project Design}

A one group pretest-posttest quasi-experimental design was used in the project to measure the impacts of assigned interventions on the key performance indicators which were selected based on possibility to be affected by the interventions after intensive literature review (Abraham et al., 2014; Bowersox, 2016; Burton et al., 2010; Colvin et al., 2016; Dimitrov and Rumrill, 2003; Mardis et al., 2017). These key performance indicators include patient satisfaction, RN (registered nurses) satisfaction, hand-Off duration, nurses practice related errors. The selected key performance indicators were measured in pre-intervention (Quarter 2) and post-intervention (Quarter 4) in 2017.

The patient satisfaction is selected to be one of the key performance indicators due to the obvious impacts of hand-off process on its results. These impacts could be as results of hand-off role in improving the patient engagement and empowerment, enhancing effective interaction and communication between nurses and patients which lead to increase the ability to identify the patients' needs correctly and completely, helping in developed based line data to monitor the patient health improvement which lead to improve patient outcome (Kullberg et al., 2017; Reimer and Herbener, 2014).

The patient satisfaction is selected to be one of the key performance indicators due to the role of hand-off process in enhancing its results and identifying the effectiveness, appropriateness, efficiency and safety of them. The patients are selected from quality management office from each department in quarterly basis. The size of sample for each unit is determined based on number of admission. At least, 30 patients are selected from the units which the number of admissions is more than 70 for each quarter. And all patients are selected from the units which the number of admissions is less than 30 .

RN satisfaction could be affected by hand-off process through its role in enhancing feeling of accountability and responsibility, performing the tasks timely and safely, organizing the tasks and assignments, and ensuring proper interaction with other nurses, patients and other health professionals (Caruso et al., 2015; Gleich et al., 2015). There is no sampling technique is conducted to select the RNs in order to access all nurses who finished the preparation period (three months), responsible for providing the care for their patient independently, and interested to participate in the survey.

The project was conducted using the lean tools which include root cause analysis, redesigning the hand-off process; standardization of hand-off process through creation required tools and checklists for a nurse to nurse hand-off, charge nurse to charge nurse hand-off and patient engagement tools for bedside hand-off; and education and training for nurses.

The root cause analysis as shown in Figure 2 highlighted that lack of standardization, a variation of nurses competencies, no clear guideline, several interruptions, every nurse endorse more than two nurses and duplication in the process are suspected to have negative impact on hand-off process.

As Figure 3 shown, redesigning the hand-off process was proposed to decrease the time consuming and removing the wastes in the hand-off process by initiating one to one hand-off between nurses' at station and bedside instead of team hand-off. Finalized by conducting the safety debriefing. The safety debriefing in the new design is used as a process to review and discuss all critical patients and issues between going 
and out coming nursing. The critical patients include the patient with chemotherapies and high alert mediation and patient for surgery or invasive procedure. Moreover, the critical issues include environmental issue, nurse manager announcement and new reported events.

\section{Data Collection}

The patient satisfaction with nursing care is reported quarterly by quality management office. The questionnaire includes 13 questions cover seven domains (respect and courtesy, patient education, patient engagement, responsiveness, active listening, safety, pain). Face validity was done for the questionnaire by sending the questions to 3 experts to confirm that the questions measured what should be measured.

Nurse satisfaction with hand-off process is measured by collecting the related data using a questionnaire. The questionnaire was developed by the project team based on extensive literature review and utilized to measure $\mathrm{RN}$-satisfaction with hand-off process in pre-intervention (Quarter 2) and post-intervention (Quarter 4). The questionnaire consists of 6 questions aimed to identify the nurses' perception regarding hand-off process including loss of information in hand-off process, effectiveness and efficiency of hand-off, feeling of accountability, safety, and errors in hand-off process, patient centeredness, and overall RN-satisfaction with hand-off process. A pilot test was conducted in one unit to confirm that the questionnaire was understandable for all nurses. Accordingly, slight modifications were done based on the staff feedback.

$\mathrm{RN}$-satisfaction questionnaires were distributed to all registered nurses in included units before and after the intervention. Face validity was done for the questionnaire by sending the questions to three experts to confirm that the questions measured what should be measure.

The duration time of hand-off was collected by filling the developed sheet by project leader to be filled by nurse managers for each shift for two weeks in the included units in pre-intervention (Quarter 2) and post-intervention (Quarter 4). A pilot test was conducted in one unit to confirm that the database was understandable and easy to use for all mangers and this test showed that there is no need for any modification.

The nurses' practices deviation related events were obtained from the nursing quality team in pre-intervention (Quarter 2) and post-intervention (Quarter 4). There are two rates were calculated: events related nurse practice deviation per 100 nurse related events, and events related nurse practice deviation per 100 reported events. These rates were selected to attain a complete picture of how conducted interventions affect the incidence of nurse practice deviation at the nursing department level as well as organization level.

\section{Ethical Approval}

The Institutional Review Board at the Center has reviewed, approved this study and granted the researchers the ethical approval number (18 KHCC 104) to conduct this study.

\section{Results}

The data was obtained in pre-intervention (Quarter 2) and post-intervention (Quarter 4) in 2017 to measure the impact of the intervention on a hand-off during, nurse satisfaction, patient satisfaction, and rate of nurse practice deviation related events.

\section{Current Process Analysis}

\begin{tabular}{|c|c|c|c|c|c|}
\hline Step & 1 & & 2 & & 3 \\
\hline Place & Station & Station & Handover Room & Station & Bedside \\
\hline Action & $\begin{array}{l}\text { Charge Nurse to } \\
\text { Charge Nurse } \\
\text { Handover }\end{array}$ & $\begin{array}{l}\text { Going } \\
\text { RNs }\end{array}$ & $\begin{array}{l}\text { Coming Charge } \\
\text { Nurse and RNs }\end{array}$ & $\begin{array}{l}\text { Going } \\
\text { RNs }\end{array}$ & $\begin{array}{c}\text { RN-RN } \\
\text { Bedside } \\
\text { Endorsement }\end{array}$ \\
\hline Waiting & All RNs Waiting & $\begin{array}{l}\text { Going RNs } \\
\text { Waiting }\end{array}$ & $\begin{array}{l}\text { Coming RNs Waiting } \\
\text { their Patients }\end{array}$ & $\begin{array}{c}\text { Going RNs } \\
\text { Waiting }\end{array}$ & Waiting \\
\hline Time & $10 \mathrm{Min}$ & & $35 \mathrm{Min}$ & & $10 \mathrm{Min}$ \\
\hline
\end{tabular}

Figue 1. Current Process

Table 1. Hand-off Duration Differences

\begin{tabular}{lcccc}
\hline & Pre-intervention & Post-Intervention & Difference & Percent Decrease \\
& Mean & Mean & & \\
\hline Duration of Hand off (Minute) & $56.1 \mathrm{~min}$ & $25.3 \mathrm{~min}$ & 30.8 & $45 \%$ \\
Nurse care duration for hand-off per day* & $8415 \mathrm{~min}$ & $3795 \mathrm{~min}$ & $4620 \mathrm{~min}$ (77 hours) & \\
& $(140.25$ hours $)$ & $(63$ hours $)$ & & \\
\hline
\end{tabular}

*Nurse hours for hand-off per shift, Number of nurses per shift * mean of duration 


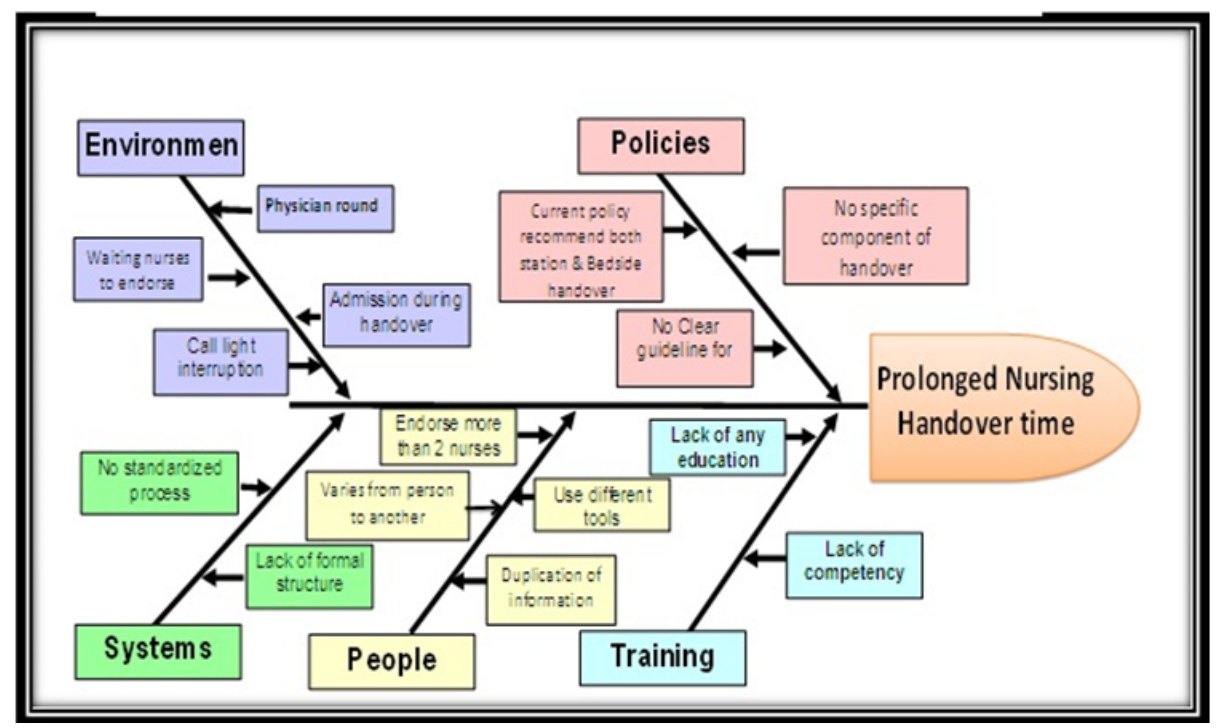

Figue 2. Root Cause Analysis

Table 2. Nurse Satisfaction with Hand-off Process

\begin{tabular}{|c|c|c|c|c|}
\hline Statement & Pre-intervention & Post-Intervention & Percent Increase & Rank \\
\hline Important patient care information is rarely lost during shift changes & $70 \%$ & $78 \%$ & $8 \%$ & 6 \\
\hline Shift changes are not problematic for patients in this hospital & $65 \%$ & $80 \%$ & $15 \%$ & 3 \\
\hline $\begin{array}{l}\text { The hand-off process might not cause any delay in providing patient } \\
\text { care }\end{array}$ & $54 \%$ & $76 \%$ & $22 \%$ & 1 \\
\hline $\begin{array}{l}\text { In the hand-off, all given patient information during the hand-off is } \\
\text { necessary and needed for me }\end{array}$ & $79 \%$ & $84 \%$ & $5 \%$ & 7 \\
\hline $\begin{array}{l}\text { The hand-off process helps RNs to prioritize patient care activities } \\
\text { and to focus on patient care needs }\end{array}$ & $76 \%$ & $88 \%$ & $11 \%$ & 5 \\
\hline $\begin{array}{l}\text { The hand-off process enable me to practice patient centered approach } \\
\text { with my patients }\end{array}$ & $72 \%$ & $87 \%$ & $15 \%$ & 3 \\
\hline I am satisfied with the hand-off process & $68 \%$ & $86 \%$ & $18 \%$ & 2 \\
\hline Total & $69 \%$ & $83 \%$ & $14 \%$ & \\
\hline
\end{tabular}

The finding of this project showed the significant decreasing in the hand-off duration post-intervention. The average duration (Pre-intervention) was 56.1 minutes, and the post-intervention was 25.3 (Table1). Moreover, the results showed that about 77 nurse care hours per shift (148 hours per day) were saved after utilizing the project.
The nurse satisfaction with the hand-off process was significantly improved. The satisfaction score was enhanced by $14 \%$ from $69 \%$ in pre-intervention to reach $83 \%$ in post-intervention. The differences between pre-intervention and post-intervention for all questions were ranged from $5 \%$ to $22 \%$. The nurse satisfaction with

\section{Targeted Condition}

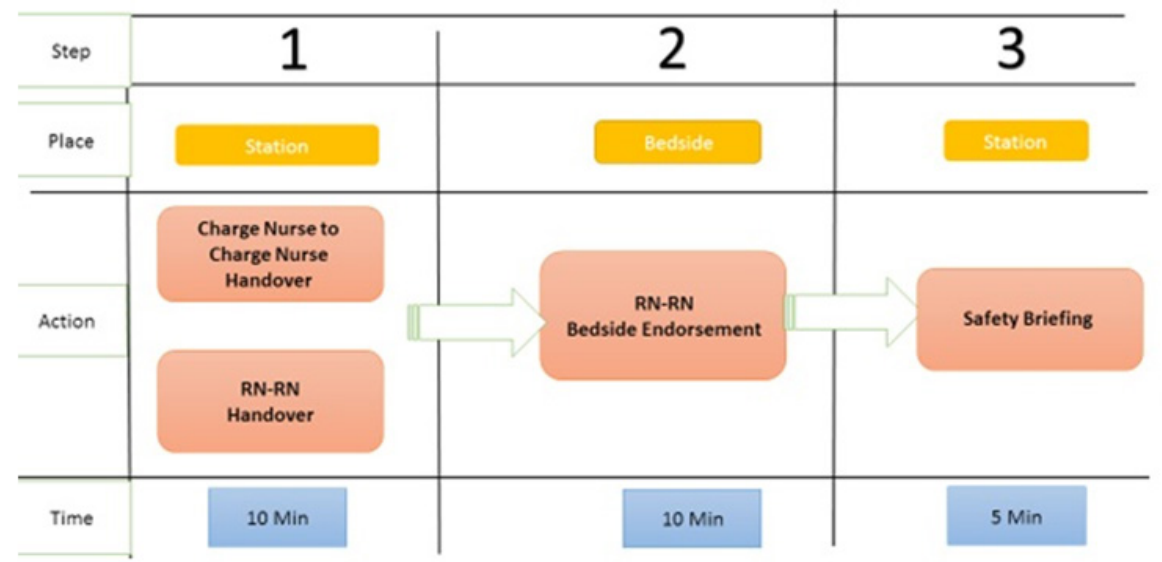

Figue 3. Targeted Condition 
Table 3. Incidence of Events Related Nurse Practice Deviation

\begin{tabular}{lccc}
\hline Events related Nurse Practice Deviation & $\begin{array}{c}\text { Pre-intervention } \\
\text { Mean }\end{array}$ & $\begin{array}{c}\text { Post-Intervention } \\
\text { Mean }\end{array}$ & Difference \\
\hline Events related Nurse Practice deviation per 100 nurse related events & 59 & 43 & 16 \\
Events related Nurse Practice deviation per 100 reported events & 36 & 22 & 14 \\
\hline
\end{tabular}

Table 4. Patient Satisfaction Results

\begin{tabular}{lccc}
\hline Patient Satisfaction Domain & Pre-intervention & Post-Intervention & Percent Increase \\
\hline Responsiveness & $90 \%$ & $91 \%$ & $1 \%$ \\
Pain management & $93 \%$ & $94 \%$ & $1 \%$ \\
Patient Education & $91 \%$ & $94 \%$ & $3 \%$ \\
Respect and Courtesy & $97 \%$ & $97 \%$ & $0 \%$ \\
Active Listening & $96 \%$ & $97 \%$ & $1 \%$ \\
Patient Engagement & $97 \%$ & $97 \%$ & $0 \%$ \\
Safety & $98 \%$ & $99 \%$ & $1 \%$ \\
Total & $95 \%$ & $96 \%$ & $1 \%$ \\
\hline
\end{tabular}

the hand-off process regarding non-causing any delay in providing patient care was the highest improved questions while the given patient information during the hand-off is necessary and needed for nurses was the lowest.

The incidence of events was also significantly decreased as shown in Table 3. The results indicated the Events related Nurse Practice deviation per 100 nurse related events and per 100 reported events was decreased from 59 to 43 (difference=16) and from 36 to 22 (difference $=14)$ respectively.

Finally, there is no significant difference in patient satisfaction results between two quarters for overall satisfaction and per each domain as shown in Table 4. The results showed that the maximum improvement was seen in patient education domain $(3 \%)$.

\section{Discussion}

This project aims to enhance the effectiveness of the hand-off process between nurses including the nurse and patient satisfaction, incidence of errors, and the duration of hand-off in the oncology setting. The project has conducted many interventions which are selected based on previous projects and studies such as standardization of hand-off process (Klee et al., 2012), structured around (Abraham et al., 2014), as well as safety debriefing (Chaboyer et al., 2010; Talleda and Hurios, 2015).

The results indicated that the adoption of lean project contributes positively in improving the effectiveness of the hand-off process between nurses in the oncology setting. The results are consistent with other lean projects which are conducted in other setting and aimed to improve cost-effectiveness and decreasing the waste of hand-off process (Aziz and Hafez, 2013; Míkva et al., 2016; Ng et al., 2010).

The results showed that adoption of the lean project in the hand-off process in oncology setting plays an important role in decreasing the duration of hand-off. This results could be induced by redesigning the process to be shorter and more effectiveness, using structured tools which contribute positively to enhancing the flow and completeness of the hand-off process. Subsequently, this leads to reduce the cost and manage the time properly as well as enhance patient and nurse satisfaction with the hand-off process. The results are consistent with other lean projects that are conducted in other setting and aimed to improve cost-effectiveness and to decrease the waste of hand-off process (Klee et al., 2012; Abraham et al., 2014).

Moreover, the results shows that the nurses indicates high satisfaction score a when implemented the structured tools and process for hand-off as results of its role in enhancing feeling of accountability and responsibility, time management, task management, and the interaction with other nurses, patients and other health professionals (Caruso et al., 2015; Gleich et al., 2015). The results are consistent with other lean projects that are conducted in other setting and aimed to improve cost effectiveness and to decrease the waste of hand-off process (Burton et al., 2010; Caruso, 2014; Caruso et al., 2015).

Additionally, the rate of nurse practice related events were significantly decreased by the standardized handoff process and tools. The results could be as results of decreasing the loss of information during the hand-off between nurses, delay in providing patient care, and increasing the nurse's ability to prioritize patient care activities and to focus on patient care needs and to practice patient-centered approach with the patients. The results consistent with other lean projects that are conducted in other setting and aimed to improve cost effectiveness and decreasing the waste of hand-off process (Abraham et al., 2012; Chu et al., 2009; Gopwani et al., 2015).

As found in previous studies, the using of the structured round can enhance patient satisfaction and engagement in the care (Brosey and March, 2015; O'Leary et al., 2016). But the results are showed that there is a slight improvement in the patient score which may be contracted with the many projects.

In conclusion the project approved that the adopting of lean principles in nursing care can enhance the safety, staff satisfaction, and effectiveness of nursing work including 
hand-off process. Moreover, it is indicated that the use of structured tools, safety briefing, and standardized hand-off process play important role in improving the effectiveness of hand-off process between nurses including staff and patient satisfaction, reducing the incidence of errors and hand-off durations, and declining the cost in oncology setting.

Accordingly, it is recommended to use management principles to improve nurses' hand-off process in oncology setting to enhance the effectiveness of nursing practice and improve nurse satisfaction. Moreover, Standardization of hand-off process by using the structured tools and conducting safety debriefing are recommended to improve staff nurses' hand-off process in oncology setting.

\section{References}

Abraham J, Kannampallil TG, Almoosa KF, Patel B, Patel VL (2014). Comparative evaluation of the content and structure of communication using two hand-off tools: Implications for patient safety. J Crit Care, 29, https://doi.org/10.1016/j. jerc.2013.11.014

Abraham J, Kannampallil T, Patel B, Almoosa K, Patel VL (2012). Ensuring patient safety in care transitions: an empirical evaluation of a Hand-off Intervention Tool. AMIA ... Annual Symposium Proceedings / AMIA Symposium. AMIA Symposium, 2012, 17-26. Retrieved from http:// www.pubmedcentral.nih.gov/articlerender.fcgi?artid=354 $0511 \&$ tool $=$ pmcentrez\&rendertype $=$ abstract.

AHRQ (2016). AHRQQ. Retrieved January 1, 2018, from https:// psnet.ahrq.gov/primers/primer/9/hand-offs-and-signouts.

Anderson J, Malone L, Shanahan K, Manning J (2015). Nursing bedside clinical handover - an integrated review of issues and tools. J Clin Nurs, https://doi.org/10.1111/jocn.12706.

Aziz RF, Hafez SM (2013). Applying lean thinking in construction and performance improvement. Alexandria Eng $J$, 52, 679-95.

Azzani M, Roslani AC, Su TT(2014). The perceived cancer-related financial hardship among patients and their families: a systematic review. Support Care Cancer, https://doi. org/10.1007/s00520-014-2474-y

Bakon S, Wirihana L, Christensen M, Craft J (2017). Nursing handovers: An integrative review of the different models and processes available. Int J Nurs Pract, https://doi.org/10.1111/ ijn. 12520 .

Bigham MT, Logsdon TR, Manicone PE, et al (2014). Decreasing hand-off-related care failures in children's Hospitals. Pediatrics, 134, 572-9.

Bowersox M (2016). A nurse's perception of hand-off communication before and after utilization of the I-5 verification of information tool. Retrieved from http://search. proquest.com/openview/70060de09cd8a98dd728f23041a11 c8f/1?pq-origsite $=$ gscholar \&cbl=18750\&diss $=y$.

Brosey LA, March KS (2015). Effectiveness of structured hourly nurse rounding on patient satisfaction and clinical outcomes. J Nurs Care Qual, 30, 153-9.

Burton MC, Kashiwagi DT, Kirkland LL, Manning D, Varkey P (2010). Gaining efficiency and satisfaction in the hand-off process. J Hosp Med, 5, 547-52.

Caruso CC (2014). Negative impacts of shiftwork and long work hours. Rehabil Nurs, 39, 16-25.

Caruso TJ, Marquez JL, Wu DS, et al (2015). Implementation of a standardized postanesthesia care hand-off increases information transfer without increasing hand-off duration. Jt Comm J Qual Saf, 41, 35-42.
Chaboyer W, McMurray A, Wallis M (2010). Bedside nursing handover: A case study. Int J Nurs Pract, 16, 27-34.

Chu ES, Reid M, Schulz T, et al (2009). A structured hand-off program for interns. Acad Med, 84, 347-52.

Colvin M, Eisen L, Gong M (2016). Improving the patient hand-off process in the intensive care unit: Keys to reducing errors and improving outcomes. Semin Respir Crit Care Med, 37, 096-106.

Costa LBM, Filho MG, Rentes AF, Bertani TM, Mardegan R (2017). Lean healthcare in developing countries: evidence from Brazilian hospitals. Int J Health Plan M, 32, 99-120.

D'Andreamatteo A, Ianni L, Lega F, Sargiacomo M (2015). Lean in healthcare: A comprehensive review. Health Policy, https://doi.org/10.1016/j.healthpol.2015.02.002

Dimitrov DM, Rumrill PD (2003). Pretest-posttest designs and measurement of change. Work (Reading, Mass.), 20, 159-65.

Fryman C, Hamo C, Raghavan S, Goolsarran N (2017). A quality improvement approach to standardization and sustainability of the hand-off process. BMJ Health Care Inform, 6 , u222156.w8291.

Gleich SL, Nemergut ME, Stans AA, et al (2015). Lean six sigma hand-off process between operating room and pediatric ICU: Improvement in patient safety, efficiency and effectiveness. Crit Care, 19, p523.

Gopwani PR, Brown KM, Quinn MJ, Dorosz EJ, Chamberlain JM (2015). Sound: A structured hand-off tool improves patient hand-offs in a pediatric emergency department. Pediatr Emerg Care, 31, 83-7.

Gupta D, Lis CG, Grutsch JF (2007). Perceived cancer-related financial difficulty: Implications for patient satisfaction with quality of life in advanced cancer. Support Care Cancer, $15,1051-6$.

Ilan R, Lebaron CD, Christianson MK, et al (2012). Handover patterns: An observational study of critical care physicians. BMC Health Serv Res, 12, https://doi.org/10.1186/14726963-12-11

Kerr D, Lu S, Mckinlay L (2014). Towards patient-centred care: Perspectives of nurses and midwives regarding shift-to-shift bedside handover. Int $J$ Nurs Pract, 20, 250-7.

Klee K, Latta L, Davis-Kirsch S, Pecchia M (2012). Using continuous process improvement methodology to standardize nursing hand-off communication. J Pediat Nurs, 27, 168-73.

Kullberg A, Sharp L, Johansson H, Brandberg Y, Bergenmar M (2017). Patient satisfaction after implementation of person-centred handover in oncological inpatient care - A cross-sectional study. PLoS One, 12, https://doi.org/10.1371/ journal.pone.0175397.

Lawal AK, Rotter T, Kinsman L, et al (2014). Lean management in health care: Definition, concepts, methodology and effects reported (systematic review protocol). Sys Rev, 3, https://doi. org/10.1186/2046-4053-3-103.

Lu S, Kerr D, Mckinlay L (2014). Bedside nursing handover: Patients' opinions. Int J Nurs Pract, 20, 451-9.

Machado Guimarães C, Crespo de Carvalho J (2013). Strategic outsourcing: a lean tool of healthcare supply chain management. Strategic Outsourcing: An Int J, 6, 138-66.

Mardis M, Davis J, Benningfield B, et al (2017). Shift-to-shift hand-off effects on patient safety and outcomes. Am J Med Qual, 32, 34-42.

Mardis T, Mardis M, Davis J (2016). Bedside shift-to-shift hand-offs: a systematic review of the literature. J Nurs Care Qual, 31, 54-60.

Míkva M, Prajová V, Yakimovich B, Korshunov A, Tyurin I (2016). Standardization-one of the tools of continuous improvement. In Procedia Engineering, 149, pp 329-32.

Ng D, Vail G, Thomas S, Schmidt N (2010). Applying the Lean principles of the Toyota Production System to reduce wait 
times in the emergency department. CA J Emerg Med, 12, $50-7$.

O'Leary KJ, Killarney A, Hansen LO, et al (2016). Effect of patient-centred bedside rounds on hospitalised patients' decision control, activation and satisfaction with care. $B M J$ Qual Saf, 25, 921-8.

Page JS, Lederman L, Kelly J, Barry MM, JamesTA (2016). Teams and teamwork in cancer care delivery: Shared mental models to improve planning for discharge and coordination of follow-up care. J Oncol Pract, 12, 1053-8.

Petersen GS, Knudsen JL, Vinter MM (2015). Cancer patients' preferences of care within hospitals: A systematic literature review. Int J Qual Health Care, 27, 384-95.

Reimer N, Herbener L (2014). Round and round we go: Rounding strategies to impact exemplary professional practice. Clin J Oncol Nurs, 18, 654-60.

Sloan T, Fitzgerald A, Hayes KJ, et al (2014). Lean in healthcare - history and recent developments. J Health Organ Manag, 28, JHOM-04-2014-0064.

Talleda J, Hurios FF (2015). Nurses' safety briefing for patient hand-off in the ICU. Revista de Enfermeria (Barcelona, Spain), 38, 48-56.

The Joint Commission (2014). Joint commission center for transforming healthcare. Retrieved January 1, 2018, from https://www.centerfortransforminghealthcare.org/assets/4/6/ hand-off_comm_storyboard.pdf.

The Joint Commission (2017). 2017 Hospital national patient safety goals. 2017 NPSG Program Links. Retrieved from https://www.jointcommission.org/assets/1/6/2017_NPSG HAP_ER.pdf.

Tirelli M, Colpa-Lewis C (2017). Nurse-led debriefing to create a culture of safety following obstetric emergencies. J Obstet Gynecol Neonatal Nurs, 3, 2.

Zapka J, Taplin SH, Ganz P, Grunfeld E, Sterba K (2012). Multilevel factors affecting quality: Examples from the cancer care continuum. J Natl Cancer Inst Monogr, 44, $11-19$.

\section{(c) (1) (9)}

This work is licensed under a Creative Commons AttributionNon Commercial 4.0 International License. 